Classification

Physics Abstracts

61.12

\title{
Nucléation homogène et cristallisation de la glace cubique (Ic) dans les verres d'électrolytes $\mathbf{L i C l} . \mathrm{D}_{2} \mathrm{O}$
}

\author{
A. Elarby, J. F. Jal, J. Dupuy \\ Département de Physique des Matériaux, LA172, 69622 Villeurbanne, France \\ P. Chieux, A. Wright \\ Institut Laue-Langevin, 156X, 38042 Grenoble Cedex, France \\ et R. Parreins
}

Laboratoire de Physique des Métaux, Université du Languedoc, 34060 Montpellier Cedex, France

(Reçu le 3 juillet 1981, révisé le 4 mars 1982, accepté le 23 mars 1982)

\begin{abstract}
Résumé. - Nous avons étudié par diffraction des neutrons, la nucléation homogène contrôlée de la glace à des températures comprises entre la transition vitreuse $T_{\mathrm{g}}=141 \mathrm{~K}$ et la température de nucléation homogène $T_{\mathrm{H}}$ dans le verre 10,5 moles $\% \mathrm{LiCl} . \mathrm{D}_{2} \mathrm{O}$. La structure obtenue est celle de la glace $\mathrm{D}_{2} \mathrm{O}$ cubique (Ic) et il n'y a pas de changement de phase apparent si l'on chauffe jusqu'à $161 \mathrm{~K}$. La structure de la matrice amorphe est conservée durant la cristallisation; on note cependant un léger déplacement des anneaux de diffusion qui est expliqué par le changement de concentration.
\end{abstract}

\begin{abstract}
A study of the controlled homogeneous nucleation of ice has been performed by neutron scattering at temperatures between the glass transition $T_{\mathrm{g}}=141 \mathrm{~K}$ and the homogeneous nucleation $T_{H}$ in 10.5 moles \% amorphous $\mathrm{LiCl} . \mathrm{D}_{2} \mathrm{O}$, as a complement to a neutron small angle scattering investigation. The diffraction pattern observed is that of $\mathrm{D}_{2} \mathrm{O}$ cubic ice (Ic). At this concentration, there is no apparent phase transformation in the crystal nuclei, nor change in the cristallization trend, as a function of the raising temperature, up to $161 \mathrm{~K}$. The amorphous phase structure is conserved during the crystallization process except for a slight peak position shift which is explained by the concentration change.
\end{abstract}

L'étude des phénomènes de nucléation dans les verres a fait d'importants progrès ces dernières années par suite de l'application de nouvelles techniques expérimentales telles que la diffusion des neutrons [1] ou les méthodes nucléaires [2] qui ont apporté une description microscopique de l'ordre à courte et à moyenne distances dans ces systèmes métastables. Ceci a été étendu à des systèmes tels que les électrolytes aqueux qui pour les fortes concentrations en soluté forment des verres à basse température [3]. Le système $\mathrm{LiCl} . n \mathrm{H}_{2} \mathrm{O}$ présentait, en particulier, la caractéristique controversée de l'existence d'une lacune de miscibilité immergée [4], dont le point critique à la concentration d'environ 12 moles $\%$ de $\mathrm{LiCl}$ dans $\mathrm{H}_{2} \mathrm{O}$ aurait été au voisinage de la température de 
transition vitreuse $T_{\mathrm{g}}$. Nous avons donc appliqué à ce problème la diffusion centrale des neutrons qui permet d'obtenir rapidement des informations sur les longueurs de corrélations des fluctuations de concentration et leur dépendance en température à l'approche d'un point critique. Les résultats de cette étude publiés séparément [5] ont mis en évidence une diffusion centrale apparaissant auprès de $T_{\mathrm{g}}$ après un temps d'incubation et pour une concentration de $10,5 \mathrm{moles} \%$ de $\mathrm{LiCl}$. Cependant cette diffusion est interprétable non pas en termes de fluctuations critiques mais comme la nucléation homogène de précipités.

Il s'avère que les valeurs respectives des températures de nucléation $\left(T_{\mathrm{H}}\right)$ et de transition vitreuse $\left(T_{\mathrm{g}}\right)$ permettent un véritable contrôle des vitesses de nucléation dans ce système. Nous représentons sur la figure 1 , le détail du diagramme des phases donné récemment par $\mathbf{C}$. A. Angell et al. [6] qui permet de bien situer notre étude. Le liquidus $\left(T_{\mathrm{L}}\right)$ du système $\mathrm{LiCl} . \mathrm{D}_{2} \mathrm{O}$ s'abaisse fortement sous l'addition de $\mathrm{LiCl}$, de même que la température de nucléation homogène en dessous de laquelle la surfusion n'est plus possible. La température de transition vitreuse par contre est pratiquement indépendante de la concentration entre $6 \%$ et environ $12 \mathrm{moles} \% \mathrm{de} \mathrm{LiCl}$

$$
\left(T_{\mathrm{g}}\left(\mathrm{D}_{2} \mathrm{O}\right) \simeq 141 \mathrm{~K}, \quad T_{\mathrm{g}}\left(\mathrm{H}_{2} \mathrm{O}\right) \simeq 139 \mathrm{~K}\right),
$$

et monte de quelques degrés seulement à plus fortes concentrations. On peut ainsi optimiser les conditions d'étude de la nucléation par le choix d'une gamme de concentrations et de températures, $T_{\mathrm{H}}>T>T_{\mathrm{g}}$, où le système sera instable par rapport à la nucléation homogène de cristaux de glace $\left(T_{\mathrm{H}}-T\right.$ indiquant le degré d'instabilité), mais suffisamment proche de $T_{\mathrm{g}}$ pour ralentir considérablement la vitesse de croissance des germes. Bien sûr aux concentrations où "l'indice de stabilité cinétique ", $\left(T_{\mathbf{H}}-T_{\mathbf{g}}\right) / T_{\mathbf{g}}$, [7] devient trop grand, le phénomène ne pourra plus être contrôlé.

Vers $10,5 \%$ de $\mathrm{LiCl}$ dans $\mathrm{D}_{2} \mathrm{O}$, les conditions de contrôle de la nucléation homogène sont très bonnes et ont pu être déterminées avec précision à partir des mesures de diffusion centrale. La

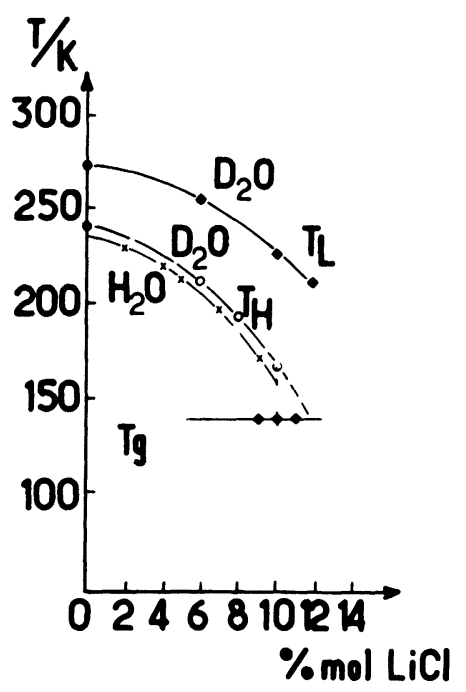

Fig. 1. - Diagramme température-concentration du liquidus $T_{\mathrm{L}}$, de la température de nucléation homogène $T_{\mathrm{H}}$, de la température de transition vitreuse $T_{\mathrm{g}}$ du système $\mathrm{LiCl} . \mathrm{D}_{2} \mathrm{O}$ (ainsi que $T_{\mathrm{H}}$ pour le système $\mathrm{LiCl} . \mathrm{H}_{2} \mathrm{O}$ ). La concentration expérimentale de notre étude est à $10,5 \mathrm{moles} \% \mathrm{de} \mathrm{LiCl}$.

[Temperature-concentration diagram of the liquidus $T_{\mathrm{L}}$, the homogeneous nucleation temperature $T_{\mathrm{H}}$, the glass transition temperature $T_{\mathrm{g}}$ of the system $\mathrm{LiCl} . \mathrm{D}_{2} \mathrm{O}$. $\left(T_{\mathrm{H}}\right.$ is also displayed for $\mathrm{LiCl} . \mathrm{H}_{2} \mathrm{O}$.) The present study is made at a concentration of 10.5 moles $\%$ of $\mathrm{LiCl}$.] 
température d'incubation est choisie à $139 \mathrm{~K}$, c'est-à-dire légèrement inférieure à $T_{\mathrm{g}}$ Au cours du réchauffement, les échantillons qui sont d'abord parfaitement transparents, deviendront successivement jaune clair, jaune orangé et blanc opaque vers $154 \mathrm{~K}$. Les tailles des cristallites ainsi que des informations sur leur distribution sont mesurées [5]. Cependant l'interprétation de la dépendance en température des résultats de diffusion aux petits angles étant délicate, on a jugé nécessaire de caractériser par diffraction de neutrons les phases présentes pendant le processus de nucléation et de cristallisation.

1. Partie expérimentale. - Nous avons effectué notre étude sur le spectromètre D2 de l'Institut Laue-Langevin à Grenoble, dans des conditions qui nous permettent d'obtenir le flux et le signal maximum $\left(\lambda=1,22 \AA\right.$, utilisation d'un petit multidétecteur de 64 cellules, collimateur $\alpha_{2}=30^{\prime}$ donnant une résolution d'appareil de $0,32^{\circ}(\Delta \theta)$, (FWHM)). En effet, nous voulons essayer d'identifier la structure des premiers stades de la nucléation et suivre son évolution au cours d'une loi de chauffe la plus proche possible de celle utilisée durant les mesures de diffusion centrale des neutrons [5], à savoir :

1) une trempe ( 10 degrés/s) à environ $100 \mathrm{~K}$ suivie d'une incubation de $41 \mathrm{~h}$ à $139 \mathrm{~K}$ (légèrement en dessous de $T_{\mathrm{g}}$ ),

2) une étude de la nucléation à $139 \mathrm{~K}(3 \mathrm{~h})$ puis à $140,4 \mathrm{~K}(14 \mathrm{~h} 30)$ (c'est à cette dernière température que dès la première mesure l'on voit commencer à poindre le signal de diffusion centrale des neutrons),

3) un retour à $139 \mathrm{~K}(3 \mathrm{~h})$ pour établir la réversibilité possible de ces premiers stades de nucléation,

4) une étude à $143 \mathrm{~K}$ ( $4 \mathrm{~h} 30$ ) (température où le signal de diffusion centrale est à un maximum),

5) un retour à $140,4 \mathrm{~K}(4 \mathrm{~h} 30)$,

6) une montée en température avec paliers : $143 \mathrm{~K}(1 \mathrm{~h}), 145 \mathrm{~K}(3 \mathrm{~h} 30), 147 \mathrm{~K}(4 \mathrm{~h} \mathrm{30}), 151,5 \mathrm{~K}$ (40 min.), $152 \mathrm{~K}(1 \mathrm{~h}), 161 \mathrm{~K}(1 \mathrm{~h})$.

A chaque température de mesure nous avons effectué un spectre assez rapide de $2<\theta<60^{\circ}$ ( $2 \theta=$ angle de Bragg) et/ou nous avons accumulé des comptages importants (environ 300000 coups/cellule) (une cellule $=0,10^{\circ}(\theta)$ ) en laissant le multidétecteur à position fixe couvrant le domaine angulaire 7,6 $\theta<13,6^{\circ}$. Cette position avait été choisie au vu des données de la structure cristalline hexagonale de $\mathrm{D}_{2} \mathrm{O}(\mathrm{Ih})$ à $5 \mathrm{~K}$ [8], qui présente un groupement de 3 pics intenses vers $10^{\circ}(\theta)$.

L'échantillon de 10,5 moles \% LiCl. $\mathrm{D}_{2} \mathrm{O}$ était dans un container en vanadium (épaisseur $0,1 \mathrm{~mm}$, diamètre $11 \mathrm{~mm}$, hauteur utile $5 \mathrm{~cm}$ ) maintenu dans un cryostat à une température régulée au $1 / 50^{\circ}$ avec une précision de $0,1^{\circ}$.

2. Résultats - Sur la figure 2 nous avons représenté quelques spectres caractéristiques. A $139 \mathrm{~K}$ après $41 \mathrm{~h}$ d'incubation et $2 \mathrm{~h}$ de mesures, le signal reste typiquement celui d'un amorphe (même à haute précision statistique, nous n'avons pas détecté d'évolution du signal). Par contre, dès que l'on passe à $140,4 \mathrm{~K}$, on commence à observer la montée d'un épaulement vers $9,5^{\circ}(\theta)$. Ceci est observable sur les comptages à haute statistique de la figure 4 et surtout sur le signal différentiel de la figure 5 , où le spectre a) représente la première détection de cet épaulement au cours de nos mesures. Cest également à cette température que l'on détecte le premier signal lors des expériences de diffusion centrale des neutrons. La question se pose de savoir si ces premiers effets observés pourraient être liés à des perturbations de l'ordre à moyenne distance dans ces systèmes; perturbations qui pourraient éventuellement être réversibles avec la température comme dans certains effets d'ordre local présentés par les amorphes [10]. Nous avons donc refroidi de nouveau le système à $139 \mathrm{~K}$ pendant $3 \mathrm{~h}$ et avons remarqué que sur toute la gamme angulaire 


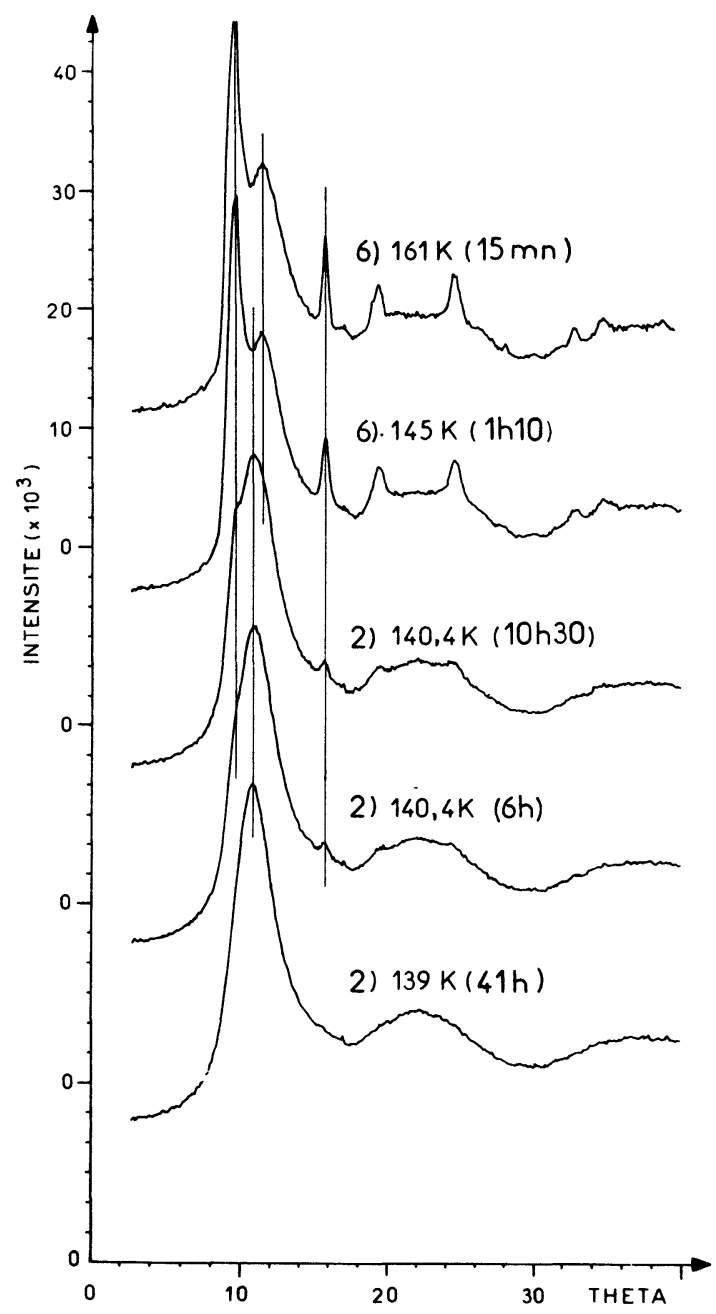

Fig. 2. - Spectres bruts de diffraction neutronique ( $2 \theta$ est l'angle de diffraction, les intensités sont en unités arbitraires). Les temps indiquent la durée de la stabilisation à la température de la mesure avant le début du spectre. Les numéros renvoient aux étapes de la procédure expérimentale.

[Neutron diffraction raw data ( $2 \theta$ is the diffraction angle, the intensities are in arbitrary units). Temperature stabilization times before initiating the runs are quoted. The numbers refer to the steps of the experimental procedure.]

d'observation le signal continuait à évoluer irréversiblement mais environ 30 fois moins rapidement qu'à $140,4 \mathrm{~K}$. Il nous faut donc attribuer l'origine du signal à $9,5^{\circ}$ à la nucléation de microcristaux $\left({ }^{1}\right)$. Si l'on chauffe maintenant à $143 \mathrm{~K}$ on note de nouveau une forte croissance du signal à $9,5^{\circ}$ (voir Fig. 6). Un refroidissement à $140,4 \mathrm{~K}$ ralentit fortement cette croissance. Enfin à plus haute température ( $145 \mathrm{~K}$ et $161 \mathrm{~K})$ (voir Figs. 2 et 3 ) de nombreux pics de la phase cristalline sont décelables, le pic à $9,5^{\circ}$ en étant le plus intense.

( $\left.{ }^{1}\right)$ Il est clair par ailleurs que les effets d'ordre à moyenne distance devraient être davantage recherchés dans la zone $0,1<q<1 \AA^{-1}(q=(4 \pi / \lambda) \sin \theta)$ c'est-à-dire dans une zone intermédiaire entre les mesures de diffusion centrale et l'étude présente, ce qui nécessite un autre équipement. 


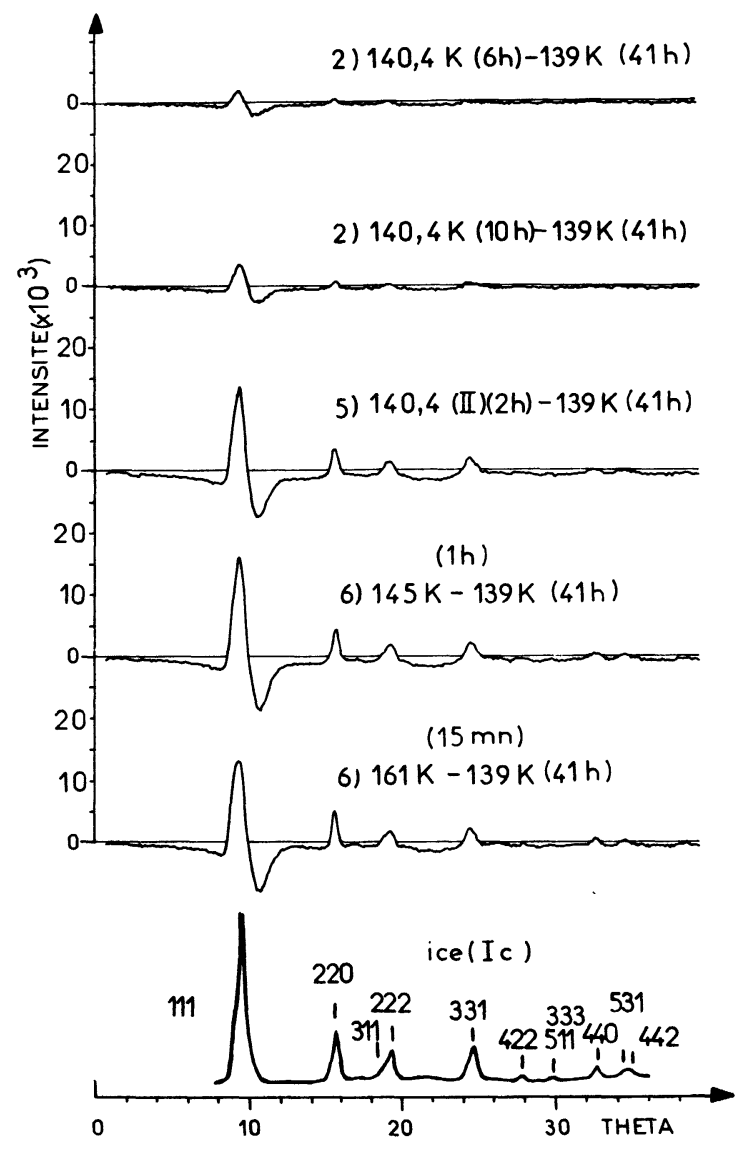

Fig. 3. - Différence de spectres entre les températures citées. Les numéros renvoient aux étapes de la procédure expérimentale. Le spectre de la glace $\mathrm{D}_{2} \mathrm{O}$ (Ic) est donné pour comparaison (voir [12]).

[Differential spectra between the printed temperatures. Stabilization times are quoted. The numbers refer to the steps of the experimental procedure. The structure of $\mathrm{D}_{2} \mathrm{O}$ ice (Ic) is given for comparison (see reference [12]).]

3. Discussion. - Notre premier objectif est d'essayer d'identifier la structure des premiers stades de nucléation aux plus basses températures où elle est décelable. En fait ceci est pratiquement impossible puisque l'intensité des pics de diffraction devient alors de plus en plus faible. La très haute précision statistique de nos mesures nous permet cependant de détecter des quantités très petites de cristaux. Et nous observons sur les figures 2 et 3 qu'il n'y a apparemment pas de changement qualitatif de structure au cours du réchauffement. Nous essayons d'identifier les pics de structure cristalline qui apparaissent (Fig. 2) en les comparant au spectre de $\mathrm{D}_{2} \mathrm{O}$ (Ih) à $5 \mathrm{~K}$ utilisé comme référence [8]. On peut dire que par rapport à cette référence il n'y a pas de pics supplémentaires, certains pics sont cependant remplacés par de faibles épaulements et de nombreux pics manquent. Le pic important vers $\theta=9,5^{\circ}$ correspondrait à 3 pics non résolus de la structure de référence $(\mathrm{Ih})$ situés à $8,98^{\circ}, 9,56^{\circ}$ et $10,19 \circ$. Cette forte disparité nous amène à envisager d'autres structures de glace, et à identifier sans ambiguité les microcristaux du précipité comme de la glace cubique (Ic) (cf. Fig. 3) [12]. Les conditions de préparation de cette glace sont assez difficiles, soit dépôt à partir de la phase vapeur sur substrat froid $(120 \mathrm{~K}-150 \mathrm{~K})$, soit pré- 


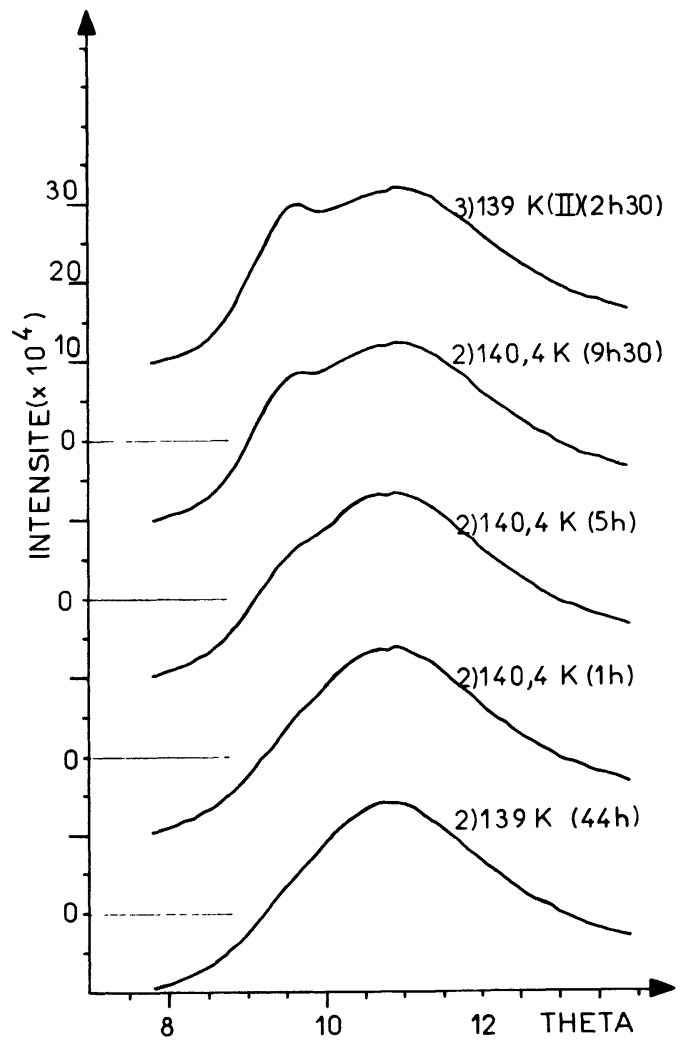

Fig. 4. - Mesures à haute précision statistique. On a indiqué les numéros de la procédure expérimentale ainsi que le temps de stabilisation avant chaque mesure.

[High precision neutron scattering curves over a narrow angular range. The temperature stabilization times are quoted. The numbers refer to the steps of the experimental procedure.]

paration à partir des phases hautes pressions de la glace trempées à l'azote et ramenées à pression atmosphérique. Ici par la trempe rapide de nos solutions nous avons peut-être une combinaison des deux procédés. Des expériences d'effet Mössbauer sur des solutions de sulfate et de chlorure de fer trempées à l'azote avaient déjà suggéré la présence de glace cubique. Ces résultats avaient cependant été contredits par des mesures de diffraction de neutrons [12] où des solutions de $\mathrm{FeCl}_{2}$ et de $\mathrm{KCl}$ dans $\mathrm{D}_{2} \mathrm{O}$ avaient systématiquement donné la structure hexagonale. Il est très possible que l'obtention de la glace cubique à partir d'une solution électrolytique soit liée aux possibilités de vitrification, i.e. aux températures $T_{\mathrm{g}}$ et $T_{\mathrm{H}}$, et à leur valeur respective par rapport aux températures de transition glace vitreuse-glace cubique-glace hexagonale [13]. Il faut remarquer que ces températures de transitions solides dépendent fortement des conditions préparatoires et de l'histoire thermique des échantillons [12,13], et donc que nos conditions actuelles de traitement thermique peuvent avoir leur importance.

Si l'on regarde maintenant la structure de la phase amorphe on remarque d'abord qu'à toute température jusqu'à $161 \mathrm{~K}$ la matrice amorphe de $\mathrm{LiCl} . \mathrm{D}_{2} \mathrm{O}$ est maintenue (cf. Fig. 2). Cependant il y a déplétion de cette phase en faveur des cristaux de glace. Ceci est particulièrement apparent sur les figures 5 et 6 où l'on voit un point isobestique caractéristique de l'échange de matière entre les deux phases. Une estimation grossière de la diminution de l'intensité cohérente sur l'ensemble de la courbe $\mathrm{LiCl} . \mathrm{D}_{2} \mathrm{O}$ amorphe, entre $139 \mathrm{~K}$ et $161 \mathrm{~K}$, nous donne une valeur de quelques \%. 


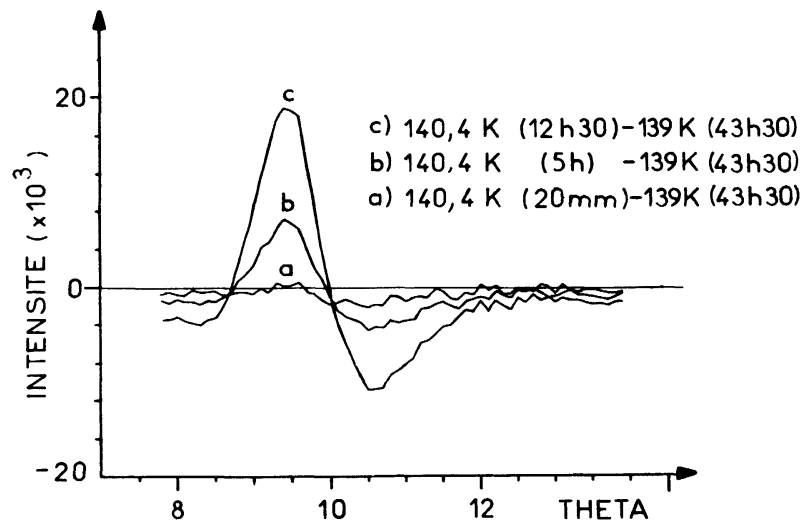

Fig. 5. - Détail des mesures différentielles de haute précision à 140,4 K. (Etape 2 de la procédure expérimentale.)

[Detail of the high precision differential spectra obtained at $140.4 \mathrm{~K}$. (Step 2 of the experimental process.)]

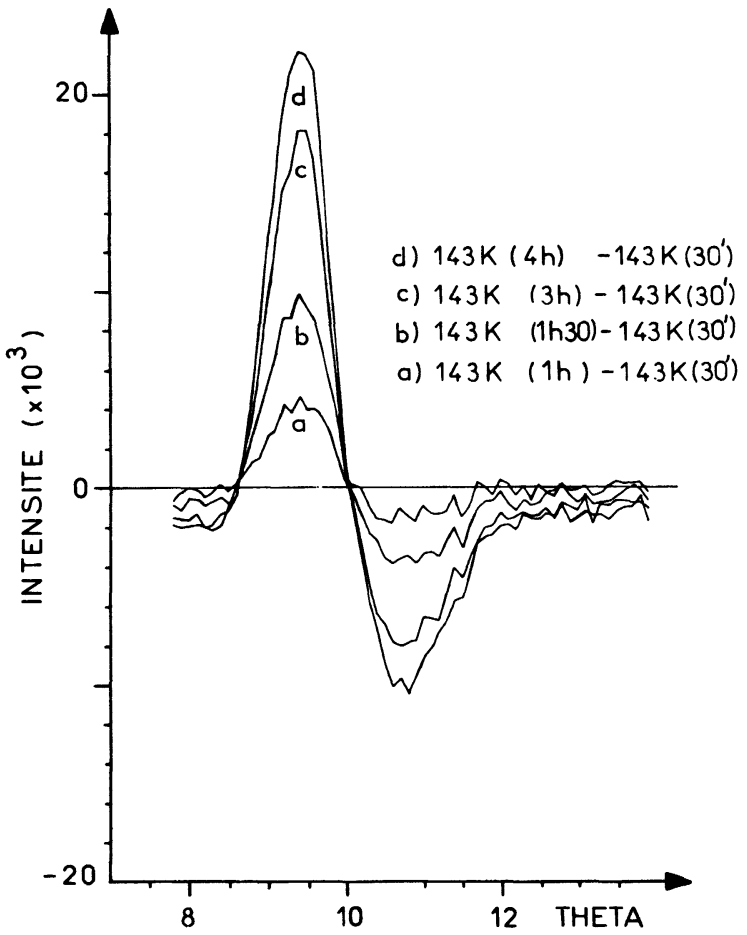

Fig. 6. - Détail des mesures différentielles de haute précision à $143 \mathrm{~K}$. (Etape 4 de la procédure expérimentale.)

[Detail of the high precision differential spectra obtained at $143 \mathrm{~K}$ by subtracting the first observed spectrum at this temperature. (Step 4 of the experimental process).]

On voit aussi que le maximum du grand anneau de diffusion de la phase amorphe se déplace de $10,8^{\circ}$ à $139 \mathrm{~K}$ à $11,4^{\circ}$ à $161 \mathrm{~K}$. Une observation détaillée de l'ensemble des spectres montre qu'il 
s'agit d'un déplacement continu de ce maximum en fonction du taux de cristallisation. L'ne explication simple en est peut-être la suivante, avec l'augmentation de concentration en $\mathrm{LiCl} . \mathrm{D}_{2} \mathrm{O}$ provoquée par la cristallisation de la glace, il y a, comme l'ont remarqué d'une façon générale Enderby et Neilson [11] pour les électrolytes aqueux près de la saturation, une inclinaison progressive des molécules d'eau sur l'axe ion-eau et donc un raccourcissement des distances iondeutérium. Cependant cette assertion demande à être vérifiée quantitativement.

Enfin quelques remarques en ce qui concerne les cristaux de glace obtenus. Nous observons l'apparition du premier signal à $9,5^{\circ}(\theta)$ à la même température $(140,4 \mathrm{~K})$ que la première observation du signal de diffusion centrale des neutrons' [5]. Ceci nous laisse supposer un lien entre les deux observations.

En effet si l'on essaye d'estimer la taille des cristallites en utilisant la formule de Scherrer [12] sur le pic 111 obtenu à $143 \mathrm{~K}$, on trouve une taille d'environ 80 à $100 \AA$, en accord avec les données de diffusion centrale qui donnent $100 \AA$ à 140,4 K. La largeur du pic est peu sensible à la température, par contre son intensité varie avec la quantité de glace précipitée. La croissance du pic 111 est environ 10 fois plus rapide à $143 \mathrm{~K}$ qu'à 140,4 K (voir Figs. 5 et 6). Le refroidissement de 140,4 à $139 \mathrm{~K}$ ou de 143 à $140,4 \mathrm{~K}$ ralentit la croissance. Dans cette gamme de températures, nous n'avons jamais observé d'arrêt ni de réversibilité des tendances à la cristallisation. Une évaluation de la quantité de glace précipitée à partir de l'intensité du pic à $9,5^{\circ}(\theta)$ mesurée à $161 \mathrm{~K}$ et normalisée par rapport à un standard de glace pure $\mathrm{D}_{2} \mathrm{O}$ (Ih) nous permet d'estimer celle-ci à une fraction volumique de l'ordre de $10 \%$. Ce qui est en accord avec la diminution apparente de l'intensité cohérente de la matrice amorphe. Ceci nous permet d'affirmer que la cristallisation commence à être détectée pour des fractions volumiques d'environ 1 à $2 \% \%^{\circ}$

4. Conclusion. - L'ensemble de nos résultats sur l'identification des phases pendant les processus de nucléation homogène et de cristallisation de la glace dans le verre $\mathrm{LiCl} . \mathrm{D}_{2} \mathrm{O}$ est donc en accord et illustre remarquablement les notions de nucléation et de croissance contrôlées par la viscosité au voisinage de $T_{\mathbf{g}}$. Nous avons de plus mis en évidence la cristallisation de la glace cubique dans la gamme de température étudiée $(139$ à $161 \mathrm{~K})$ pour la concentration de 10,5 moles \% LiCl. $n \mathrm{D}_{2} \mathrm{O}$. Nous n'avons pas encore précisé la température de transition entre la glace cubique et la glace hexagonale, dans notre système mais normalement celle-ci a lieu vers $190 \mathrm{~K}$ et ne devrait pas perturber notre étude. Ce point mérite cependant une investigation.

Il n'y a pas de modification sensible de la structure de la phase amorphe au cours de la nucléation de la glace. Un léger déplacement de l'anneau de diffusion est sans doute dû à un effet indirect de concentration qu'il faudra vérifier quantitativement. Nous pensons que ces résultats nous permettront d'interpréter l'ensemble de nos mesures de chaleur spécifique, transmission de la lumière et diffusion centrale des neutrons [5]. En effet, par ces diverses techniques les premiers signaux obtenus après traitement thermique identique, s'observent toujours aux mêmes températures et ceci est en parfait accord avec l'apparition de la nucléation de la glace détectée dans l'étude présente. Cependant dans le cas de la diffusion centrale des neutrons une décroissance importante du signal a été notée à quelques degrés au-dessus de $T_{\mathrm{g}}$ [5]. Cette décroissance pouvait avoir pour origine soit une diminution du nombre de cristallites par suite de réarrangements locaux soit une perte de contraste neutronique purement fortuite, liée à la variation de densité de la matrice vitreuse. Nos mesures de structure confirment que les cristaux de glace continuent à se former dans cette région. Il n'y a pas de diminution apparente du signal de diffraction et rien ne permet de laisser supposer une variation dans la répartition de la taille des cristaux. Ceci est en accord avec l'interprétation donnée aux résultats macroscopiques de conductibilité électrique obtenus par ailleurs [9].

Remerciements. - Nous tenons à remercier MM. J.-L. Soubeyroux et P. George pour l'aide apportée au cours des mesures à l'ILL. 


\section{Bibliographie}

[1] Wright, A. F., Talbot, J., Fender, B. E. F., Nature 277 (1979) 366.

[2] Rapport R.C.P. Liquides et Amorphes, Nancy, Oct. 1980.

[3] Angell, C. A., Sare, E. S., J. Chem. Phys. 52 (1970) 1058.

[4] Hsih, H., Gammon, R. W., Macedo, P. B., Montrose, C. J., J. Chem. Phys. 56 (1972) 1663.

[5] Ferradou, C., Jal, J. F., Calemezuk, R., Parreins, R., Angell, C. A., Wright, A., Dupuy, J., to appear in Nature.

[6] Angell, C. A., Sara, E. J., Donnella, J., MacFarlane, D. R., J. Phys. Chem. 85 (1981) 1461.

[7] Angell, C. A., Clarke, J. H. R., Woodcock, L. V., Adv. Chem. Phys. 48 (1981) 398.

[8] Lehmann, M., (Private Communication).

[9] Angell, C. A., MacFarlane, D. R., to appear in J. Phys. Chem.

[10] Balanzat, E., Mairy, C., Hailleret, J., J. Physique Colloq. 41 (1980) C8-871.

[11] Enderby, J. E., NeILson, G. W., in Water, a Comprehensive Treatise (Ed. F. Franks) 1979, Vol. 6.

[12] Arnold, G. P., Finch, E. D., Rabideau, S. W., Wenzel, R. G., J. Chem. Phys. 49 (1968) 4365.

[13] Hoвbs, P., Ice Physics, University of Washington (Clarendon Press, Oxford) 1974, p. 45-53. 